\title{
Specific Heat of Para-Hydrogen Monolayers on Graphite*
}

\author{
Michael Bretz \\ Department of Physics, University of Michigan, Ann Arbor, Michigan $\dagger$ \\ and University of Washington, Seattle, Washington \\ and T. T. Chung \\ Department of Physics, University of Washington, Seattle, Washington
}

(Received June 7, 1974)

The specific heat of para-hydrogen films has been measured from 1 to $20 \mathrm{~K}$ for areal densities between 0.01 and 0.055 atoms $/ \AA^{2}$ of the partial monolayer adsorbed on grafoil. The results are compared with the specific heat of ${ }^{4} \mathrm{He}$ films at equivalent densities. It is argued that a nonideal gas regime and liquefaction are present in both systems and that solidification of the $\mathrm{p}-\mathrm{H}_{2}$ films has been suppressed to below the temperature range of the experiment. Implications regarding $2 D$ superfluidity in hydrogen monolayers are discussed briefly.

Recent studies exploring the character of partial monolayer helium films adsorbed on a graphite substrate have discovered and examined in detail a rich variety of existing monolayer phases. ${ }^{1}$ The results have attracted keen theoretical interest in the role of dimensionality in quantum phase transitions $\mathrm{s}^{2,3}$ and have renewed speculation on the possibility of $2 \mathrm{D}$ remnant Bose condensation in helium films. ${ }^{4}$ Molecular hydrogen was chosen for the present investigation because its light mass and strong polarizability should provide important and intriguing variations in the behavior of quantum monolayer films. Interpretations are straightforward since the molecule of hydrogen is approximately spherical (atomic centers are $0.74 \AA$ apart), physisorbs via van der Waals forces onto graphite, and quickly converts to the spin- 0 (para) state once on the substrate.

This paper reports specific heat data taken for para-hydrogen films physisorbed on graphite at low monolayer coverages. We used a standard grafoil-filled calorimeter of $108 \mathrm{~cm}^{3}$ STP ${ }^{4} \mathrm{He}$ monolayer capacity which was charged with a $\sim 90 \%$ para-hydrogen mixture. The gas was admitted with the

*Work supported in part by the National Science Foundation.

$\dagger$ Permanent address.

(c) 1974 Plenum Publishing Corporation, 227 West 17th Street, New York, N.Y. 10011. No part of this publication may be reproduced, stored in a retrieval system, or transmitted, in any form or by any means, electronic, mechanical, photocopying, microfilming, recording, or otherwise, without written permission of the publisher. 


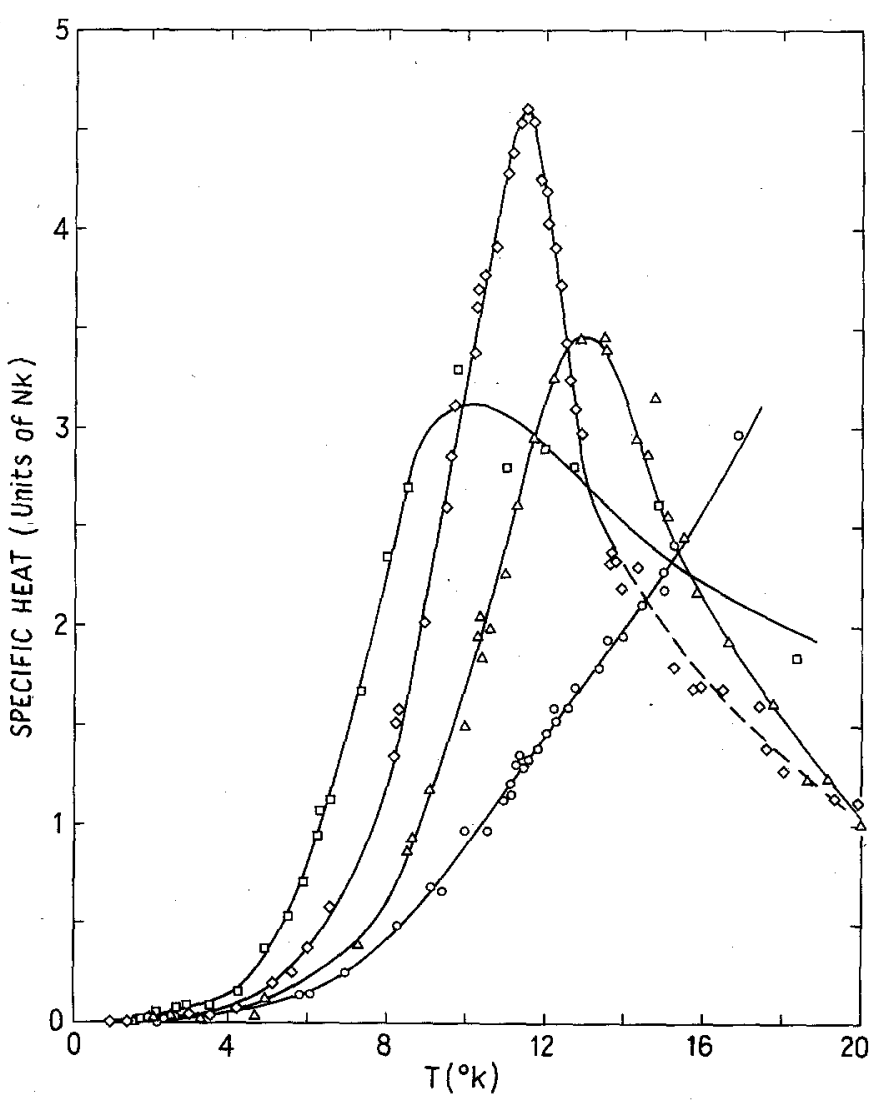

Fig. 1. Specific heat of $p-\mathrm{H}_{2}$ submonolayers. Densities are calculated assuming the same monolayer adsorption area as for ${ }^{4} \mathrm{He}$ films adsorbed on graphite. ${ }^{1}$ Values of $n:(\square) 0.0112 ;(\diamond) 0.0277 ;(\triangle) 0.0409$; (O) 0.055 .

cell, ${ }^{4} \mathrm{He}$ pot, and fill line above $50 \mathrm{~K}$ and cooled over an interval of a few days to ensure uniform substrate plating. Data-taking was delayed another few days until residual ortho-para conversion died out (as monitored by any temperature rise in the isolated calorimeter). All samples were reheated midway through data acquisition to check for proper annealing. Riehl and Fisher, ${ }^{5, *}$ who made NMR measurements on partial monolayers of orthohydrogen physisorbed on graphitized carbon black, found a signal decay of $\leq 5 \% / \mathrm{h}$ below $30 \mathrm{~K}$, evidently due to ortho-para conversion. They obtained reproducible results with $\frac{1}{2}-1 \mathrm{~h}$ cooldown from $77 \mathrm{~K}$, which indicates that the procedures used above are entirely adequate for uniform plating and conversion to para-hydrogen. Adequate isotherms for para-hydrogen on

*Conclusions from the NMR experiments should not be directly compared with the present results since ortho- and para-hydrogen have quite dissimilar properties. 
graphite are unfortunately not available so the present analysis incorporates the helium film values of $108 \mathrm{cc}$ STP monolayer capacity and 0.11 atoms $/ \AA^{2}$ at monolayer completion. ${ }^{1}$

Results for the four coverages investigated are displayed in Fig. 1. For the lowest coverage, $n=0.0112$ molecules $/ \AA^{2}$, the specific heat signal appears quadratic in $T$ below $4 \mathrm{~K}$. This quickly grows into a broad maximum near $10 \mathrm{~K}$, and although the data are sketchy at higher temperature, it seems to drop to values substantially above $C / N k=1$ at $20 \mathrm{~K}$. Higher coverages display the same quadratic low-temperature dependence and specific heat peak. But the peaks are considerably sharper and are displaced toward higher temperatures, the $n=0.055$ maximum finally rising above the temperature range of the experiment. Although the data extend only to $20 \mathrm{~K}$, it appears that the curves for $n=0.027$ and 0.0409 will undershoot $C / N k=1$, the classical gas value.

Figure 2 reproduces some previous specific heat results for monolayer ${ }^{4} \mathrm{He}$ films at areal densities $n$ near those obtained for hydrogen. ${ }^{1}$ On comparing Figs. 1 to 2, it is apparent that, except for scale factors, the hydrogen features correspond closely to those for helium at corresponding monolayer densities. We can qualitatively explain the scale factors which give the

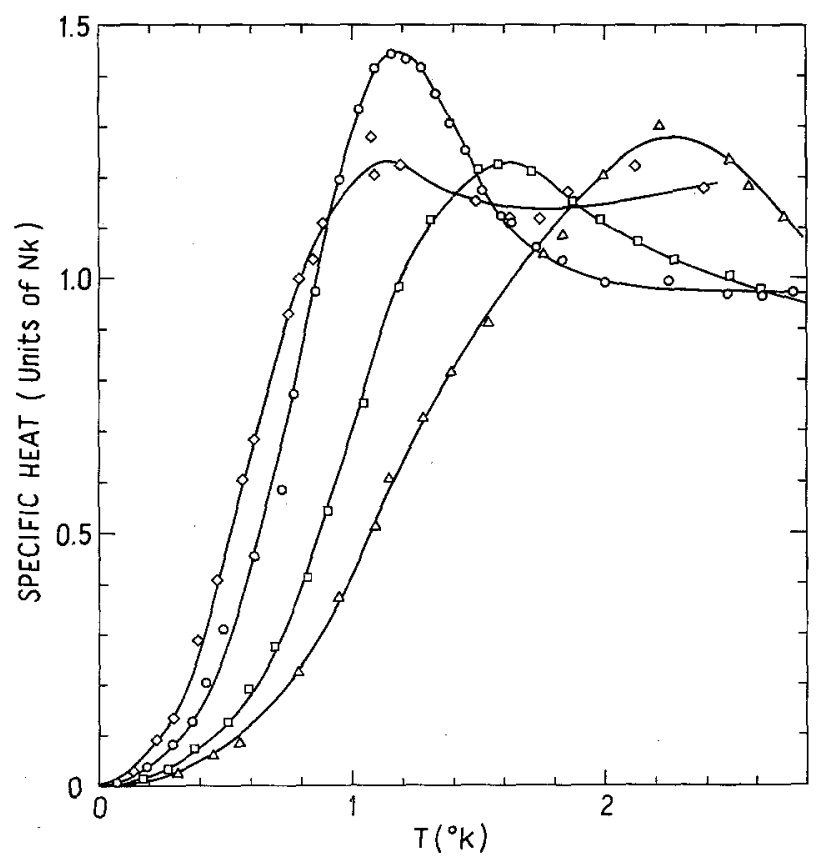

Fig. 2. Specific heat of ${ }^{4} \mathrm{He}$ submonolayers adsorbed on graphite; reproduced from Ref. 1 . Values of $n\left(\right.$ atom $\left./ \AA^{2}\right):(\diamond) 0.0165 ;(O)$ $0.0280 ;(\square) 0.042 ;(\triangle) 0.0484$. 
significantly higher temperature and specific heat values of $\mathrm{H}_{2}$ over ${ }^{4} \mathrm{He}$. Interactions between classical atoms or molecules are usually approximated by the Lennard-Jones potential

$$
\varphi=4 \varepsilon\left[(\sigma / r)^{12}-(\sigma / r)^{6}\right]
$$

where the energy parameter $\varepsilon$ and distance parameter $\sigma$ are specified for the particular substances (see Table I). The classical law of corresponding states gives reduced state variables

$$
P^{*}=P \sigma^{3} / \varepsilon, \quad V^{*}=V / N \sigma^{3}, \quad T^{*}=k T / \varepsilon
$$

and the partition function $z=f\left(V^{*}, T^{*}\right)$, where $f$ is a universal function for all systems. $f$ has been modified to $f\left(V^{*}, T^{*}, \Lambda^{*}\right)$ by DeBoer ${ }^{6}$ to account for quantum effects through the parameter $\Lambda^{*}=h / \sigma(m \varepsilon)^{1 / 2}$, where $h$ is Planck's constant and $m$ is the molecular mass. The $\Lambda^{*}$ correction allows for the depressed bulk critical temperatures and increased critical volumes of the light, highly quantum mechanical noble gases relative to those for the heavier, more classical gases. We might expect the $\mathrm{H}_{2}$ film specific heat peaks to occur at considerably higher temperatures than for the ${ }^{4} \mathrm{He}$ peaks. For if these two-dimensional peak temperatures $T_{p}$ scale as the bulk critical temperatures, then

$$
\frac{T_{p}^{\mathrm{H}}}{T_{p}^{\mathrm{He}}}=\frac{T_{\left(\Lambda^{*}\right)}^{* \mathrm{H}}}{T_{\left(\Lambda^{*}\right)}^{* \mathrm{H}} \frac{\varepsilon^{\mathrm{H}}}{\varepsilon^{\mathrm{He}}}}=\frac{T_{c\left(\Lambda^{*}\right)}^{* \mathrm{H}}}{T_{c\left(\Lambda^{*}\right)}^{* \mathrm{He}}} \frac{\varepsilon^{\mathrm{H}}}{\varepsilon^{\mathrm{He}}}=\frac{0.688}{0.390} \frac{37}{10.2}=6.4
$$

This ratio approaches the observed scale factor of about 9 between the corresponding peaks of Figs. 1 and 2. The ratio of areal densities is also calculated. We see that the zero-point contributions implicit in $V_{\left(\Lambda^{*}\right)}^{*}$ approximately cancel the fractional volume charge of the hard cores.

$$
\frac{n^{\mathrm{He}}}{n^{\mathrm{H}}} \simeq\left(\frac{V^{\mathrm{H}}}{V^{\mathrm{He}}}\right)^{2 / 3}=\left(\frac{V_{\left(\star^{*}\right)}^{* \mathrm{H}}}{V_{\left(\Lambda^{*}\right)}^{* \mathrm{He}}}\right)^{2 / 3}\left(\frac{\sigma^{\mathrm{H}}}{\sigma^{\mathrm{He}}}\right)^{2}=\left(\frac{1.39}{1.90}\right)^{2 / 3}\left(\frac{2.93}{2.56}\right)^{2}=1.06
$$

One might argue that the partition function $f$ gives only a crude representation for the properties of a film adsorbed onto a real substrate where

TABLE I

Lennard-Jones Parameters, Quantum Parameter $\Lambda^{*}$, and Effects of $\Lambda^{*}$ on the Classical Reduced Critical Points $T_{c}^{*}$ and $\boldsymbol{V}_{c}^{*}$ for Various Molecules ${ }^{6}$

\begin{tabular}{cccccc}
\hline Molecule & $\varepsilon / k, \mathrm{~K}$ & $\sigma, \AA$ & $\Lambda^{*}$ & $T_{c}^{*}\left(\Lambda^{*}\right) / T_{c}^{*}$ & $V_{c}^{*}\left(\Lambda^{*}\right) / V_{c}^{*}$ \\
\hline $\mathrm{Ne}$ & 35.6 & 2.75 & 0.59 & 0.955 & 1.07 \\
$\mathrm{H}_{2}$ & 37.0 & 2.93 & 1.73 & 0.688 & 1.39 \\
${ }^{4} \mathrm{He}$ & 10.2 & 2.56 & 2.68 & 0.390 & 1.90 \\
${ }^{3} \mathrm{He}$ & 10.2 & 2.56 & 3.08 & 0.252 & 2.30 \\
\hline
\end{tabular}


periodic variations, inhomogeneities, etc. modify the adatom behaviorespecially for high- $\varepsilon$-parameter atoms like hydrogen. However, as we shall discuss shortly, qualitative agreement does exist between the high-temperature hydrogen film data and a sophisticated calculation which assumes a perfectly uniform substrate. In like spirit, then, the law of corresponding states should be acceptable as a guide to an understanding of the temperature shift of the peaks.

Simple entropy considerations explain the large specific heat values observed for hydrogen films. Both ${ }^{4} \mathrm{He}$ and $\mathrm{H}_{2}$ will asymptotically approach classical 2D gas behavior at high temperatures, where the entropy per particle is $2+\ln \left(2 \pi m k T / n h^{2}\right)$. Since $s=\int(C d T / N k T)$ a small ${ }^{4} \mathrm{He}$ peak near $1.4 \mathrm{~K}$ contributes about as much to the total entropy as does a large $\mathrm{H}_{2}$ peak at 10-14 K (numerical entropy values will be presented later).

Now that the scaling between Figs. 1 and 2 is qualitatively understood, it is appropriate to inquire whether the insights gained through extensive analysis of the helium film data can be used to interpret the hydrogen film behavior as well. At the lowest helium coverages $\left(\lesssim 0.02 / \AA^{2}\right)$ the heat capacity peaks are weak and the $4 \mathrm{~K}$ values are near $C / N k=2$. These results are presently understood in terms of disrupted film behavior due to preferential adsorption on the few inhomogeneous regimes of the graphite substrate (e.g., edgeplanes, impurities, dislocations). ${ }^{1,3}$ Hydrogen films should also show some heterogeneity at the lowest coverage and the large $15-20 \mathrm{~K}$ specific heat for $n=0.0112 / \AA^{2}$ is undestood on this basis.

At somewhat greater densities where inhomogenieties are unimportant the high-temperature (near $4 \mathrm{~K}$ ) signature for helium films is that of a 2D gas. In this region the specific heat of a $2 \mathrm{D}$ quantum virial gas can be expanded as

$$
C / N k=1-n \beta^{2}\left(d^{2} B / d \beta^{2}\right)+O\left(n^{2}\right)
$$

where $\beta=1 / k T$ and $B$ is the second virial coefficient.* Siddon and Schick ${ }^{7}$ have calculated $B$ and its derivatives using the Lennard-Jones 6-12 interaction with the parameters for helium and the assumption of a smooth substrate. These results match in detail the specific heat rise with decreasing temperature on the high- $T$ side of the peak for both ${ }^{4} \mathrm{He}$ and ${ }^{3} \mathrm{He}$ (not shown in Fig. 2). The success of these calculations, using only the second virial coefficient, enable higher-order terms in $n$ to be dropped so that $(C / N k-1) / n$ can be set equal to $-\beta^{2} d^{2} B / d \beta^{2}$, which is a constant independent of $n$ for all coverages. The hydrogen specific heat data above $15 \mathrm{~K}$ for $n=0.0409$ in Fig. 1 and the above relationship between coverage and $C / N k$ [Eq. (1)] have been used to estimate the specific heat for $n=0.0277$. The calculated signal for $n=0.0277$, given as the dashed line in Fig. 1, is within the experimental scatter of the data.

*We ignore possible rotational effects, which are exponentially small at the temperatures of this experiment. See Ref. 8 for further details. 


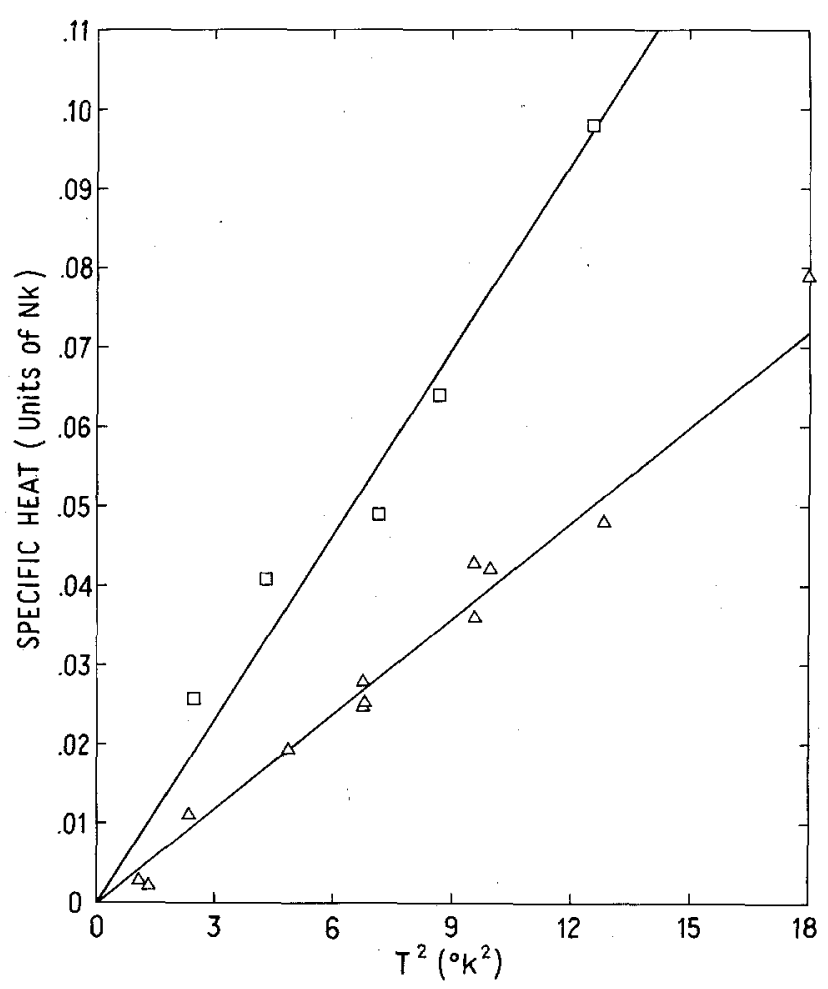

Fig. 3. $C / N k$ plotted vs. $T^{2}$ for the two lowest coverages $n=0.0122$ ( $\square$ ) and $n=0.0277(\triangle)$. The solid lines are visual fits to the data.

This indicates that the second-order virial expansion is sufficient to describe the high-temperature hydrogen data. In the following paper ${ }^{8}$ Siddon and Schick have extended their 2D virial calculation to $p-\mathrm{H}_{2}$ monolayer films where an increase in the specific heat with decreasing temperature is also predicted. Although their analysis qualitatively explains the high-temperature hydrogen data, quantitative agreement is lacking, for reasons discussed in their paper. It must also be noted that the ratios of experimental heat capacity signals for charged to empty calorimeter are within $10 \%$ of unity at temperature approaching $20 \mathrm{~K}$. Thus, existing background uncertainties of $1-2 \%$ in this region due to reduced calorimeter isolation are greatly magnified and could significantly displace the reduced specific heat data.

From a plot of $B$ vs. $\beta$ and its relation to the van der Waals imperfect gas law constants Siddon and Schick ${ }^{8}$ have estimated the critical temperature and density of liquefaction in $2 \mathrm{D}$ parahydrogen films. Their values, $T_{c}=8.4 \mathrm{~K}$ and $n_{c}=0.012 / \AA^{2}$, are close to the observed peak temperatures and densities of Fig. 1. However, we must discuss another possibility-that of sublimation 
between 2D nonideal gas and solid phases-before concluding that 2D liquefaction is actually being observed. Resolution of this question requires an analysis of the low-temperature specific heat data. Figure 3 gives the specific heat plotted vs. $T^{2}$ for $n=0.0112$ and $n=0.0277$ where sufficient low-temperature data were taken to make a meaningful analytic fit. Both curves vary as $T^{2}$ over a range of several degrees. Debye $\theta$ 's have been calculated and are given in Table II along with estimates of $\theta_{\mathrm{D}}$ for the higher coverages where only sparse data exist. The melting temperature $T_{m}$ of any 2D solid can be determined simply from its Debye temperature using the expression $^{3}$

$$
T_{m}=\left[1 /(2 \pi)^{2}\right]\left(m k_{\mathrm{B}} / 8 \hbar^{2} n\right) \theta_{\mathrm{D}}^{2}
$$

Values of $T_{m}$ have been estimated using the $\theta_{\mathbf{D}}$ values from Table II and the apparent film density $n$. These melting temperatures range from 30 to $60 \mathrm{~K}$, which is considerably above the observed peak temperatures of Fig. 1. Conversely, taking the $T_{m}$ 's as the experimental values, estimates of film density in the solid phases are calculated. These densities (Table II) are very large, and except for the lowest coverage film, are close to the monolayer completion density of $0.11 / \AA^{2}$. For comparison, the $2 / 3$ power of the bulk density of hydrogen at $13.8 \mathrm{~K}, \rho_{\text {bulk }}=0.023 / \AA^{3}$, is calculated and multiplied by a geometric factor of 1.091 for conversion from the $3 \mathrm{D}$ hcp to the $2 \mathrm{D}$ triangular close-packed configurations. ${ }^{3}$ The resulting areal density, $0.074 / \AA^{2}$, is comparable to $n_{m}=0.0674$ of the solid phase in the $n=0.0112$ film and considerably below the solid density $n_{m}$ calculated for the other coverages. 2D films have a smaller number of nearest neighbors than in 3D although the van der Waals attractive forces are to first approximation unaffected by dimensionality. Any solid monolayer phase not subject to the high pressure present near monolayer completion should be significantly less dense than the corresponding 3D solid because of the reduced number of nearest neighbors, not $1 \frac{1}{2}$ times bulk areal density which would be the case if we

TABLE II $^{a}$

\begin{tabular}{crrl}
\hline Coverage, $\AA^{-2}$ & $\theta_{\mathrm{D}}, \mathrm{K}$ & \multicolumn{1}{c}{$T_{m}, \mathrm{~K}$} & \multicolumn{1}{c}{$n_{m}$} \\
\hline 0.0112 & 61 & 60 & 0.0674 \\
0.0277 & 86 & 48 & 0.117 \\
0.0409 & $\sim 92$ & $\sim 38$ & $\sim 0.12$ \\
0.055 & $\sim 100$ & $\sim 33$ & $\sim 0.11$ \\
\hline
\end{tabular}

${ }^{a}$ Debye $\theta$ 's are calculated from Fig. 3 using $C / N k=28.8\left(T / \theta_{\mathrm{D}}\right)^{2}$, the expression for the specific heat of a $2 \mathrm{D}$ solid with $T \ll \theta_{\mathrm{D}}$. The $T_{m}$ values are calculated from Eq. (2) using coverages $n$ and the $\theta_{\mathbf{D}}$ 's given. Conversely, $n_{m}$ is the density of the solid phase found from Eq. (2) with the peak temperature of Fig. 1 used for $T_{m}$. 


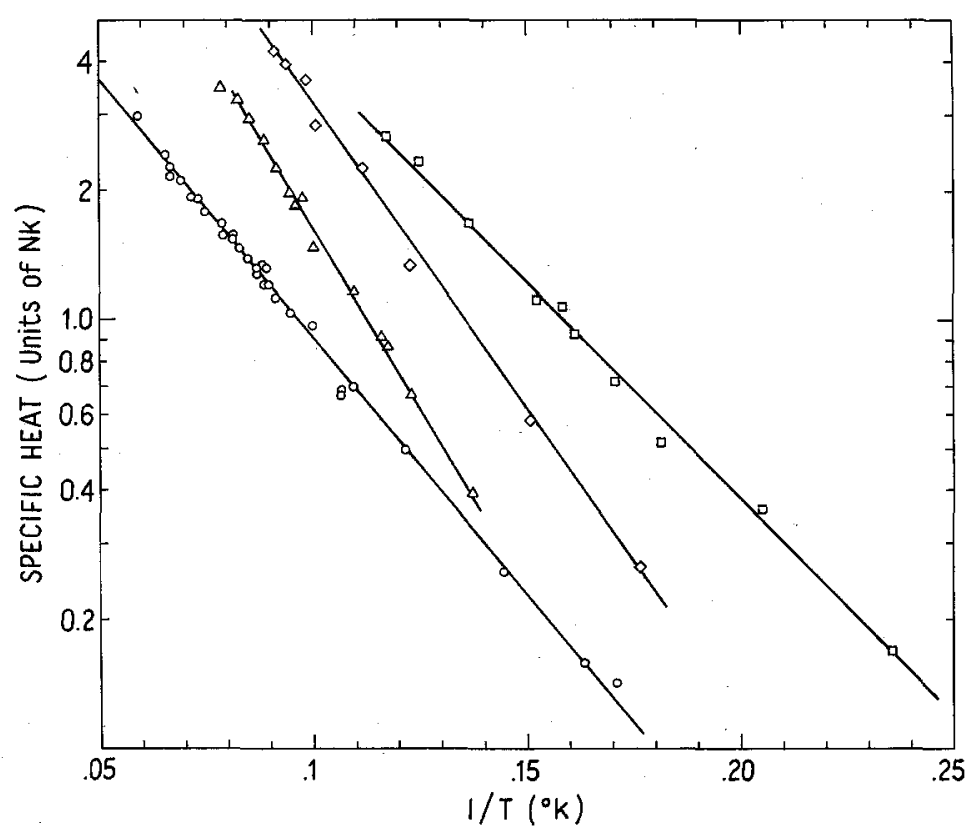

Fig. 4. $\log C / N k$ plotted vs. $1 / T$ showing the exponential specific heat behavior. Solid lines are visual fits to the data whose parameters are: $C / N k=$ $37.5 e^{-23.0 / T}$ for $n=0.0112(\square) ; 85.3 e^{-32.8 / T}$ for $n=0.0277(\diamond) ; 87.8 e^{-39.75 / T}$ for $n=0.0409(\triangle)$; and $15 \cdot 34 e^{-28.4 / T}$ for $n=0.055(O)$.

believed the $n_{m}$ values of Table II. It is apparent, then, that the peaks of Fig. 1 are not due to sublimation between nonideal gas and 2D solid phases.

Substrate-mediated effects such as a localized-mobile transition or narrow tunneling bands are also ruled out. A recent heat capacity study of neon on graphite in the temperature range $1-20 \mathrm{~K}$ found no evidence for tunneling phenomena for this nearly classical adsorbent. ${ }^{9}$ Moreover, it is well known from pressure isotherms that classical noble gases are highly mobile on graphite, passing through a succession of phases with increasing coverages. ${ }^{10}$ Thus, it is unlikely that hydrogen, with a large $\Lambda^{*}$ value, would become localized.

Rounding of the peak for both helium and hydrogen films can be understood as a temperature smearing caused by either surface inhomogeneities ${ }^{2}$ or phase equilibrium problems. ${ }^{*}$ For temperatures below the peak and above the $T^{2}$ region all coverages clearly follow an exponential dependence in $1 / T$. The exponential fit, which can provide additional information regarding the properties of the condensed film phase, is in contrast to the ${ }^{4} \mathrm{He}$ data of Fig. 2, where no such dependence is found. The slopes of the

*The latter was suggested by J. G. Dash (see Ref. 7). 
straight lines drawn through the data of Fig. 4 are the film activation energies. Since we believe the films to be in the $2 \mathrm{D}$ liquid state, these activation energies are proportional to the latent heats of $2 \mathrm{D}$ vaporization $l$. Values of $l / k$ are given in the legend to Fig. 4, along with the proportionality constants $C_{0} / N k$. The latent heats increase with coverage. This trend, along with the increase in $T_{\text {peak }}$ with $n$, is consistent with a rising spreading pressure $\varphi$ in the 2D vapor phase. The largest coverage $n=0.055$, however, has a considerably lower latent heat and proportionality constant $C_{0} / N k$ than the other films. This is evidence that the atoms are finding new energy configurations in the film. We believe that the anomalous behavior of the $n=0.055$ hydrogen film is almost certainly caused by the approach to registry with the substrate wells. Helium films on graphite undergo an order-disorder transition at epitaxy ( $n=0.0637$, where one helium atom is present for every 3 graphite potential wells). ${ }^{1}$ Although hydrogen films were only investigated at lower densities, a similar transition in hydrogen films is expected.

The specific heat multiplied by $1 / T$ was integrated from 0 to $20 \mathrm{~K}$ for the coverage $n=0.0277$ to determine the specific entropy of the film. The functional fit $C / N k=0.0040 T^{2}$ was used below $4.5 \mathrm{~K}, 85.3 e^{-32.8 / T}$ was taken up to $11 \mathrm{~K}$, and a graphical integration was made from 11 to $20 \mathrm{~K}$. The resulting entropy, $s=1.99$, is well below the ideal $2 \mathrm{D}$ gas value at $20 \mathrm{~K}$, $S / N k=2+\ln \left(2 \pi m k T / n h^{2}\right)=3.28(m$ is molecular mass and $n$ is the areal density). A further entropy contribution arises from the non-ideality of the 2D vapor above $20 \mathrm{~K}$. This has been estimated from the hydrogen virial coefficients ${ }^{8}$ to be $S / N k \sim 0.4$, bringing the resulting entropy up to ${ }^{11} 2.4$. It is clear from this comparison that a significant fraction of the total film entropy is missing, and a glance at Fig. 1 will confirm that the $20 \mathrm{~K}$ entropy value decreases with increasing coverage. A further film regime with a specific heat well above the $T^{2}$ fit must be present below $1 \mathrm{~K}$ to account for the missing entropy.

Recently Ginzburg and Sobyanin ${ }^{12}$ pointed out that a superfluid phase of molecular hydrogen would become a distinct possibility if the bulk melting temperature could be suppressed somehow to below $\sim 6 \mathrm{~K}$. They specifically mentioned that the wide range of densities accessible for adsorbed films on graphite could be utilized to force a large reduction in the melting temperature of physisorbed hydrogen (melting point at $1 \mathrm{~atm}$ is $13.8 \mathrm{~K}$ in bulk). It appears that solidification has been suppressed to below $1 \mathrm{~K}$ for the coverages of the present experiment. However, as with helium monolayers, the heat capacity signature of a $2 \mathrm{D}$ superfluid phase transition is not presently known, so the monolayer hydrogen data of Fig. 1 will not tell us if $2 \mathrm{D}$ superfluidity exists in these films. Techniques for probing transport properties on individual crystallites must be utilized or developed for this purpose. 
In summary, the behavior of $p-\mathrm{H}_{2}$ films at partial monolayer coverages is surprisingly similar to that of ${ }^{4} \mathrm{He}$ films at comparable densities. A nonideal 2D gas is present at the highest temperatures and liquefaction probably takes place as the temperature is lowered. The exponential specific heat signature indicates that this liquefaction occurs via a first-order transition. Since solidification has not been detected within the temperature range of this experiment, the presence of $2 \mathrm{D}$ superfluid hydrogen remains an intriguing and important possibility. Entropy considerations suggest further thermodynamic activity below $1 \mathrm{~K}$ (e.g., solidification, localization, superfluidity) so the exploration of $p-\mathrm{H}_{2}$ films at very low temperatures should be productive.

\section{ACKNOWLEDGMENTS}

We wish to thank G. B. Huff for technical guidance in use of the cryostat and Jim Goodwin for assistance in data acquisition. Discussions with M. W. Cole, J. G. Dash, L. M. Sander, M. Schick, and R. L. Siddon were most helpful.

\section{REFERENCES}

1. M. Bretz, J. G. Dash, D. C. Hinkernell, E. O. McLean, and O. E. Vilches, Phys. Rev. A 8, 1589 (1973).

2. A. D. Novaco, J. Low Temp. Phys. 9, 457 (1972).

3. R. L. Elgin and D. L. Goodstein, Monolayer and Submonolayer Helium Films, Daunt and Lerner, eds. (Plenum Press, New York), p. 35.

4. C. E. Campbell, J. G. Dash, and M. Schick, Phys. Rev. Letters 26, 963 (1971); A. Widom and J. B. Sokoloff, Phys. Rev. A 5, 475 (1972).

5. J. W. Riehl and C. J. Fisher, J. Chem. Phys. 59, 4336 (1973).

6. J. DeBoer, Physica 14, 139 (1948). (Also see pages 315 and 510 of this volume.)

7. R. L. Siddon and M. Schick, Phys. Rev. A 9, 907 (1974).

8. R. L. Siddon and M. Schick, following paper in this issue.

9. G. B. Huff, "Neon Adsorbed on Exfoliated Graphite. A Heat Capacity and Vapor Pressure Study," Ph.D. Thesis, Univ. of Washington (1972), unpublished.

10. A. Thomy and X. Duval, J. Chim. Phys. 67, 1101 (1970).

11. T. M. Sanders, private communication.

12. V. L. Ginzburg and A. A. Sobyanin, Soviet Phys.-JETP Letters 15, 242 (1972). 